\section{e0255 RELATIONSHIP OF ALCOHOL CONSUMPTION AND CAROTID ATHEROSCLEROSIS IN CHINESE HAN UIGHUR AND HAZAKH COHORT}

doi:10.1136/hrt.2010.208967.255

Xie Xiang, Ma Yitong, Yang Yining, Fu Zhenyan, Ma Xiang, Huang Ding, Li Xiaomei, Chen Bangdang, Liu Fen. Department of Cardiovascular Medicine, The First Affiliated Hospital Xinjiang Medical University

Aim The relationship between alcohol consumption and carotid atherosclerosis had been reported in some epidemiologic studies. But the results were conflicting in different researches. In the present study, we investigated the association between alcohol intake and carotid atherosclerosis in Chinese Han, Uighur, and Hazakh population.

Methods and Results The study population sample comprised 13037 Chinese people (5277 Han, 4572 Uighur, and 3188 Hazakh) aged 35 years and over, who participated a Cardiovascular Risk Survey between June 2007 and September 2009. Daily alcohol consumption was determined by the number and frequency of alcoholic beverages consumed. The carotid artery parameters including common carotid artery intima-media thickness (CCA-IMT) and carotid plaques were measured using high-resolution B-mode ultrasonography. In Han or Hazakh, carotid IMT as a function of alcohol consumption was depicted as a J-shaped curve with a nadir for the alcohol intake category of 20 to $29.9 \mathrm{~g} / \mathrm{d}$; In Uighur, the similar curve with a nadir of 30 to $49.9 \mathrm{~g} / \mathrm{d}$ was observed. For the prevalence of carotid plaques, we also observed the similar curves in Han and Hazakh but not in Uighur. After adjusted for the age, sex, blood pressure, body mass index, smoking, GLU, total cholesterol, HDL, and LDL, the J-shaped curves remain existence.

Conclusions Our results indicated that moderate drinking is a protective factor for carotid atherosclerosis. But the definition of moderate drinking should be difference in Han, Uighur and Hazakh population.

\section{e0256 ALCOHOL CONSUMPTION AND ANKLE-TO-BRACHIAL INDEX}

doi:10.1136/hrt.2010.208967.256

Xie Xiang, Ma Yitong, Yang Yining, Huang Ding, Li Xiaomei, Ma Xiang, Chen Bangdang, Liu Fev. Department of Cardiovascular Medicine, The First Affiliated Hospital Xinjiang Medical University

Aim A low ankle-to-brachial index (ABI) is a strong correlate of cardiovascular disease and subsequent mortality. The relationship between $\mathrm{ABI}$ and alcohol consumption remains unclear.

Methods and Results Data are from the Cardiovascular Risk Survey (CRS), a multi-ethnical community-based study of 14593 Chinese people (5749 Han, 4747 Uighur, and 4097 Hazakh) aged 35 years and over at baseline in June 2007 to March 2010. The relationship between alcohol intake and ABI were determined by use of analysis of covariance and multivariable regressions. In men, a linear correlation between alcohol consumption and $\mathrm{ABI}$ was found by one-factor analysis of variance $(p<0.001)$; After adjusted for the age, sex, ethnicity, blood pressure, body mass index, smoking, GLU, total cholesterol, HDL, and LDL, the difference remains significant $(p=0.007)$. The multivariate-adjusted $O R$ for peripheral artery disease was significantly higher in men who consumed $>60.0 \mathrm{~g} / \mathrm{d}$ $(\mathrm{OR}=1.997,95 \% \mathrm{CI} 1.500$ to 2.989$)$ and was significantly lower in men who consumed $20.0-40.0 \mathrm{~g} / \mathrm{d}$ compared with never drinking, respectively ( $p=0.01, p=0.027$, respectively, data not shown). Neither $\mathrm{ABI}$ nor $\mathrm{PAD}$ was correlated with alcohol intake in women.

Conclusions Our results indicated that in Chinese men, moderate drinking is a protective factor but heavier drinking is a risk factor for peripheral arteriosclerosis.

\section{E0257 ASSOCIATION OF C REACTIVE PROTEIN LEVELS WITH THE SEVERITY OF CORONARY ARTERY DISEASE}

doi:10.1136/hrt.2010.208967.257

${ }^{1}$ Liu Haihang, ${ }^{1}$ Zhao Dong, ${ }^{2}$ Ma Changsheng, ${ }^{2}$ Liu Xiaohui, ${ }^{2}$ Lv Qiang, ' $\mathrm{Ci}$ Yue, ${ }^{1}$ Li Yan ${ }^{1}$ Ren Jie, 'Liu Jing. ${ }^{1}$ Capital Medical University, Affiliated Beijing Anzhen Hospital, Beijing Institute of Heart Lung and Blood Vessel Diseases; ${ }^{2}$ Capital Medical University, Affiliated Beijing Anzhen Hospital

Objective To explore the association of CRP levels with the severity of coronary stenosis in patients with coronary artery disease (CAD) documented by angiography.

Methods A total of 368 patients with angiographically determined $\mathrm{CAD}$ (defined as stenosis of $>=50 \%$ in 1 or more coronary arteries) were enrolled after exclusion of patients with acute myocardial infarction, acute infectious diseases or CRP $>=10 \mathrm{mg} / \mathrm{l}$, and chronic liver or kidney diseases. Serum CRP was measured using particle enhanced immunoturbidimetric method (DiaSys, Germany). CAD severity was assessed by the number of stenotic coronary arteries and the Gensini score. In the current study, LDL-C $<130 \mathrm{mg} / \mathrm{dl}$ was defined as the lower level of LDL-C and LDL-C $3130 \mathrm{mg} / \mathrm{dl}$ as the higher level of LDL-C.

Results Of 368 patients, 179 patients had single-vessel stenosis, 105 had 2 stenotic vessels, and 84 had $\geq 3$ stenotic vessels. Systolic blood pressure and triglycerides levels increased significantly with the number of stenotic arteries. Median and inter-quartile range (IOR) of CRP in patients with single-vessel stenosis and multi-vessel stenosis was $0.93(0.44-2.41) \mathrm{mg} / \mathrm{l}$ and $1.33(0.66-2.39) \mathrm{mg} / \mathrm{l}$, respectively, $p=0.030$. Univariate analysis found that participants with CRP $\geq 1 \mathrm{mg} / \mathrm{l}$ had a significantly higher Gensini score (29.0 $(12.0-56.0)$ vs $20.0(10.0-46.2), p=0.026)$ and higher prevalence of multi-vessel stenosis $(57.6 \%$ vs $44.1 \%, \mathrm{p}=0.010)$ than those with CRP $<1 \mathrm{mg} / \mathrm{l}$. After age, gender, body mass index, systolic blood pressure, smoking status, fasting glucose, HDL-C and LDL-C adjustments, CRP levels remained to be associated with CAD severity. The OR was 1.77 (95\% CI 1.14 to 2.76) for patients with a higher level of CRP ( $\geq 1 \mathrm{mg} / \mathrm{l})$ versus those with a lower level of CRP $(<1 \mathrm{mg} / \mathrm{l})$. Among patients with a lower level of LDL-C, treated or not treated with statins therapy, the prevalence of multivessel stenosis was higher in those having a higher level of CRP than in those having a lower level of CRP ( $55.0 \%$ vs $41.7 \%, p=0.024)$. Further analysis was undertaken by dividing the patients into 4 categories according to CRP levels and whether or not they had statins treatment. Compared with the risk of CAD in patients with a lower level of CRP who were taking statins, the risk increased significantly when the CRP level was higher, regardless of whether they were taking statins or not. The risk was the highest $(\mathrm{OR}=2.15$, 95\% CI 1.08 to $4.27, p=0.029$ ) for those with a higher level of CRP but who were not on statins therapy.

Conclusion CRP is associated with the severity of CAD. It may provide additional information regarding the risk of presenting multi-vessel stenosis even in patients with lower LDL-C.

\section{e0258 CURRENT CLINICAL PRACTICE AND GUIDELINE APPLICATION IN THERAPIES OF ST SEGMENT ELEVATION ACS INPATIENT IN A MULTI-PROVINCIAL STUDY IN CHINA RESULTS OF BRIG PROJECT}

doi:10.1136/hrt.2010.208967.258

Guo Lai-Jing, Wang Jiansong, Zhao Dong, Liu Jing, Liu Jun, Zhang Huiying, Xing Liying, Sun Jiayi, Wang Miao. Chronic Disease Institute1, Beijing University Shougang Hospital

Objective To evaluate the current practice of medications that have been proven effective by evidence-based medicine on inpatients with ST segment elevation ACS in China. 